\title{
Transaction
}

\section{Mechanism of Water- and Oil-Repellency Behavior of Paper Prepared by Internal Addition of Fluorine-Containing Reagent}

\author{
Satoru Fukuda ${ }^{{ }_{1}}$, Akira Isogai ${ }^{* 1}$, Fumihiko Onabe ${ }^{{ }^{* 1}}$, Masahiko Ishikawa ${ }^{* 2}$, and Tetsuya Masutani ${ }^{* 2}$ \\ ${ }^{* 1}$ Graduate School of Agricultural and Life Sciences, The University of Tokyo, \\ 1-1-1 Yayoi, Bunkyo-ku, Tokyo 113-8657, Japan \\ ${ }^{* 2}$ Daikin Industries Ltd., 1-1 Nishi Hitotsuya, Settsu-shi, Osaka 566-8585, Japan
}

\begin{abstract}
Handsheets were prepared from pulp slurries by internal addition of a water-soluble fluorochemical (fluoroalkylphosphate diethanolamine salt) with or without polyamideamine-epichlorohydrin resin (PAE), and water- and oilrepellency behavior of the handsheets was studied in terms of handsheet-making conditions. The micro-flask combustion method and X-ray fluorescence analysis were used to determine fluorochemical contents in the handsheets. The fluorochemicals were retained in the handsheets by electrostatic interactions through PAE, in which case high degrees of water- and oil-repellency were given to the handsheets. Nearly no fluorochemicals were retained in the handsheets without PAE. In most cases, water-repellency of the handsheets was sensitively influenced by the handsheet-making conditions, whereas oil-repellency was roughly governed by the fluorochemical contents in the handsheets. The key factors for improvement of water- and oil-repellency may lie not only in retaining the fluorochemicals but also in controlling distribution or agglomeration of the fluorochemicals in the handsheets.
\end{abstract}

(Received 5 November, 2001; Accepted 18 January, 2002)

\section{Introduction}

Fluorochemicals generally exhibit quite small critical surface energy, and diminish contact angles against liquids so that they have particular properties of repellency against not only water but also oil. If these reagents are retained in paper properly, then both water- and oilrepellency are expected to take effect on the paper. Thus, paper, paperboard and pulp mold prepared with fluorochemicals may widely be used for wrapping and packaging of foods, machines coated with oil and so on, partly in place of the conventional packages made of nonbiodegradable synthetic polymers.

In comparison with paper coated with pigments containing fluorine-containing reagents, internal addition of these reagents will give liquid-repellency to paper and paperboard more effectively with smaller addition levels of the reagents. Moreover, paper and paperboard prepared by internal addition will show stable liquid-repellency in contrast to coated paper even after cutting or folding process. On the other hand, however, retention behavior of the fluorochemicals added at the wet-end must be sufficiently understood for controlling white water circulation and pitch troubles.
So far, only a few papers about coating of fluorochemicals on base paper have been reported [1], and there has been no report dealing with internal addition. In this paper, handsheets were prepared under various conditions by internal addition of a water-soluble fluorine-containing reagent, and retention mechanism was studied in terms of handsheet-making conditions. Furthermore, factors influencing virtual water- and oil-repellency behavior of the handsheets were investigated from several aspects.

\section{Experiment}

\subsection{Materials for making handsheets}

The fluorine-containing reagent (abbreviated hereafter as F-reagent) used in this study has the chemical structure of difluoroalkylphosphate diethanolamine salt $(\mathrm{n}=$ $2,3,4,5,6$, on the average $\mathrm{n}=$ ca. 3.5; TG-810, Daikin Industries Ltd., Japan), and is mostly soluble in water. A representative chemical structure of the F-reagent is shown in Fig. 1. The perfluoroalkyl groups in the molecules show liquid-repellency. A polyamideamineepichlorohydrin resin (PAE; WS-570, Japan PMC Co., Japan) was used as a cationic retention aid. A commercial 


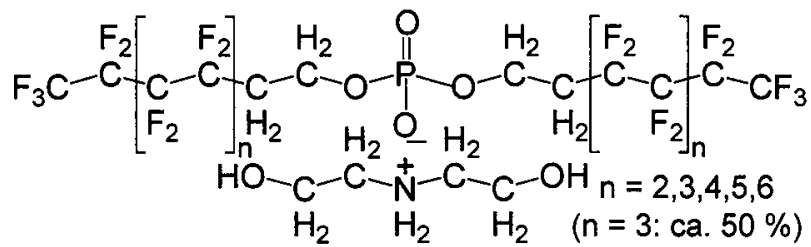

Fig. 1 Representative chemical structure of the fluorine-containing reagent used in this study.

bleached hardwood kraft pulp was used for handsheet-making after beating to $550 \mathrm{~mL}$ C.S.F. with a PFI mill.

\subsection{Methods of papermaking}

To a $0.15 \%$ pulp slurry, $0.3 \%$ (on dry weight of pulp) PAE and the F-reagent of $0-1.2 \%$ (on dry weight of pulp) were added in this sequence. After all additives were added, the pulp slurry was stirred for $1 \mathrm{~min}$ before being subjected to handsheet-making [2]. After couching and wet-pressing, the wet sheets were dried at $23^{\circ} \mathrm{C}$ and $60 \%$ R.H. (or at $20^{\circ} \mathrm{C}$ and $65 \%$ R.H. for some handsheets) for 1 day. Some handsheets were then heated at $105^{\circ} \mathrm{C}$ for $30 \mathrm{~min}$ in an oven, followed by conditioning again at $23^{\circ} \mathrm{C}$ and $60 \%$ R.H. (or at $20^{\circ} \mathrm{C}$ and $65 \%$ R.H. for some handsheets) for more than 1 day. In several cases, these conditions about the addition amount and the sequence of additives, time of stirring the pulp slurry before handsheet-making and the heating temperature of the handsheets were modified.

\subsection{Analysis of water- and oil-repellency of handsheets}

Stöckigt sizing degree [3] was adopted to evaluate water-repellency of the handsheets. Oil-repellency of the handsheets was evaluated by "Kit Test" [4] and expressed as the value from 0 to 12 .

\subsection{Determination of F-reagent content in handsheets}

The F-reagent contents in the handsheets were determined by the micro-flask combustion method [5]. In this method, the handsheet sample attached to platinum holder was burned in the atmosphere of oxygen in a Schöniger combustion flask with sodium peroxide (Wako Pure Chemical Industries, Ltd., Japan) as a promoter of combustion. Then the F-reagent components in the handsheet sample were converted to fluorine ions and became soluble in water present in the flask. After $30 \mathrm{~min}$, the water containing $\mathrm{F}^{-}$ions thus formed in the flask was mixed under a ratio of $1: 1$ with TISAB solution, which was a buffer solution for $\mathrm{F}^{-}$ionic strength, made from acetic acid, sodium hydroxide and trisodium citrate dihydrate (Wako Pure Chemical Industries, Ltd., Japan) [5]. Then $\mathrm{F}^{-}$ions in the solution were determined using a selective electrode system that consisted of a series of $F^{-}$ ion selective electrode, reference electrode and ion meter (F-125, HS-305DP and IM-5S, respectively DKK-TOA Corporation, Japan). The F-reagent contents were.calculated from the fluorine contents in the handsheet samples on the basis of the chemical structure of the F-reagent with $n=3.5$ in Fig. 1 .

Along this flask combustion method, X-ray fluorescence method was adopted as a subsidiary method for determination of the F-reagent content in the handsheets. In this method phosphorus contents due to the F-reagent present in the handsheets were measured using an X-ray fluorescence analyzer (MESA 500; Horiba Co., Japan) and the F-reagent content in the handsheets was relatively estimated. Samples of the handsheets were irradiated for $100 \mathrm{~s}$ with $\mathrm{X}$-ray generated from an X-ray tube at a tube voltage of $15 \mathrm{kV}$ and a tube electric current of $500 \mu \mathrm{A}$. Then fluorescent X-ray launched on the samples was detected with a Si-detector and the pulses output from it were counted at $2.01 \mathrm{keV}$.

\section{Results and discussion}

\subsection{Determination of the F-reagent content in handsheets by $\mathrm{X}$-ray fluorescence analysis}

As to all the handsheets presented in this study except those in Fig. 7, peak intensity of fluorescent X-ray due to phosphorus was measured with $\mathrm{X}$-ray fluorescence analyzer and the F-reagent contents in the handsheets were determined with this method. The correlation between the

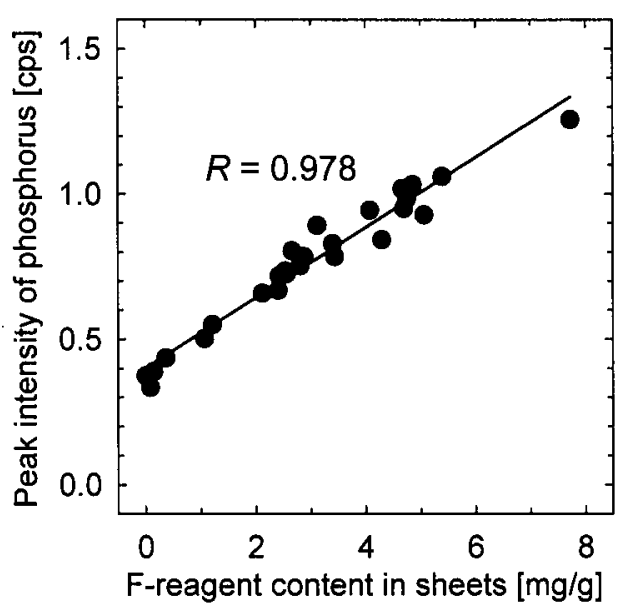

Fig. 2 Correlation between content of F-reagent and peak intensity of phosphorus in X-ray fluorescence analysis of handsheets. Correlation function is $p=0.1207 f+0.4011$, where $p$ and $f$ are peak intensity of phosphorus of handsheets [cps] and F-reagent content [mg/g], respectively. 
phosphorus peak intensities and the F-reagent contents measured by the flask combustion method was noted (Fig. 2). Correlation coefficient $R$ was 0.978 , and therefore appropriate correlation was obtained. From this result, $\mathrm{X}$-ray fluorescence analysis, which does not destroy samples and is simple and easy to operate, is considered to be an appropriate method for rough determination of the F-reagent content in the handsheets although the flask combustion method was mainly adopted on this study for more precise determination.

\subsection{Liquid-repellency and the F-reagent content determined by the micro-flask combustion method of handsheets}

Fig. 3 shows the relationships between the F-reagent addition level in the handsheet-making process and either degree of liquid-repellency or the F-reagent content of the handsheets. The F-reagent components were barely retained in the handsheets, when they were prepared without PAE as a cationic retention aid. This implies that nearly no F-reagent components could be retained in the handsheets only by physical filtration effects or van der Waal's interactions. In other words, the F-reagent having anionic charges due to the dissociated phosphate groups must be retained in the handsheets by electrostatic interactions between the anionic F-reagent molecules (or colloids in part) and anionic pulp fibers through cationic retention aids in the pulp slurry.
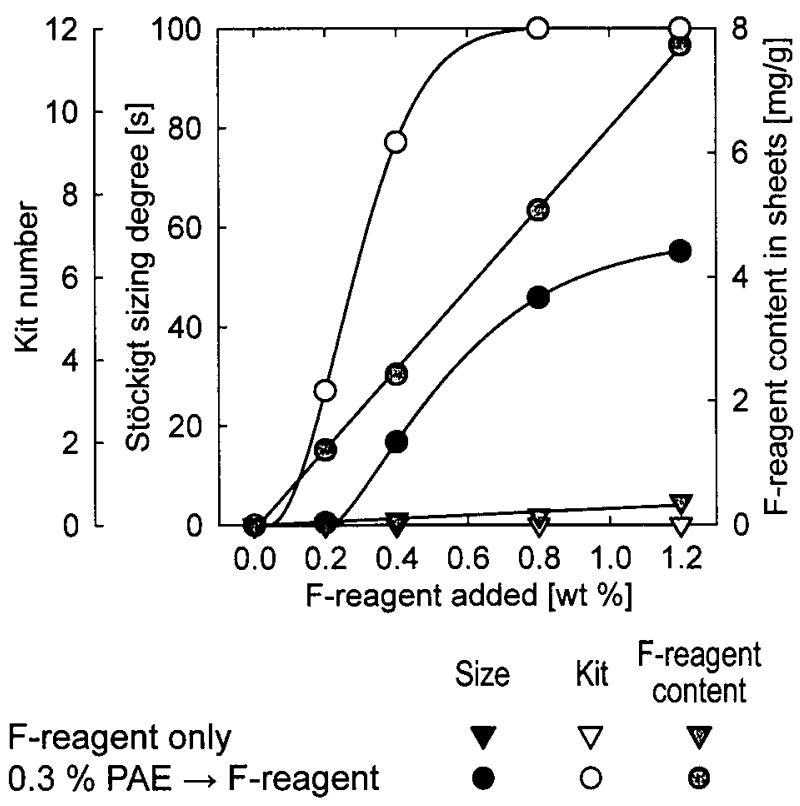

Fig. 3 Water- and oil-repellency and F-reagent content of the handsheets prepared with or without PAE. The handsheets were prepared after stirring the slurry for $1 \mathrm{~min}$, and heated at $105^{\circ} \mathrm{C}$ for $30 \mathrm{~min}$ after conditioning.

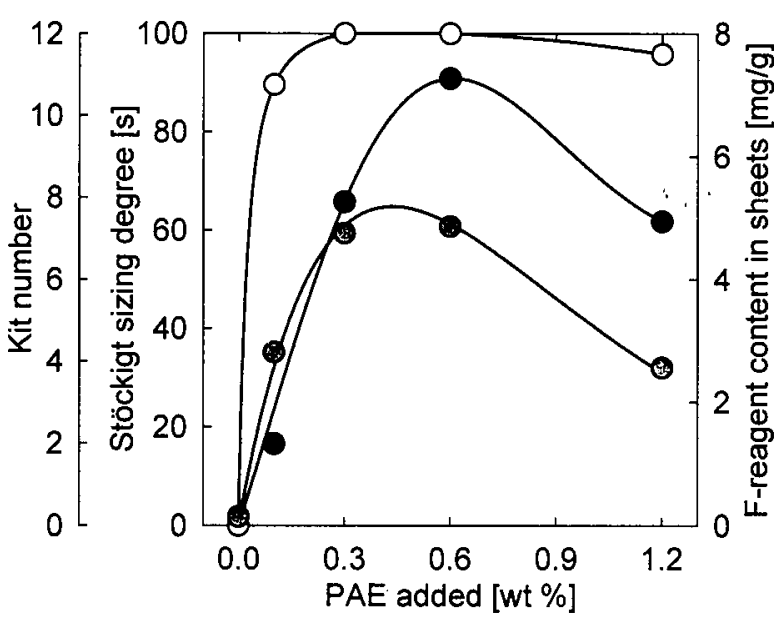

Size: Kit:O F-reagent content:-

Fig. 4 Water- and oil-repellency and F-reagent content of the handsheets prepared with $0-1.2 \% \mathrm{PAE} \rightarrow$ $0.8 \%$ F-reagent. The handsheets were prepared after stirring the slurry for $1 \mathrm{~min}$ and heated at $105^{\circ} \mathrm{C}$ for $30 \mathrm{~min}$ after conditioning.

The F-reagent content in the handsheets increased with increasing the F-reagent addition level, and retention ratios of the F-reagent (the amounts of the F-reagent retained in the handsheets divided by those added to the pulp slurry) did not fall in spite of the rise in addition level: approximately around $60 \%$.

\subsection{Influences of the amount of PAE added to pulp slurry}

Variations in the addition sequence of the F-reagent and PAE as a cationic retention aid had some influence on the retention of the F-reagent as well as the resultant liquid-repellency. For instance, when the amount of PAE was changed from $0.3 \%$ to $0.1,0.6$ and $1.2 \%$, water-repellency and the F-reagent content of the handsheets were predominantly affected (Fig. 4). On the other hand, oil-repellency was already induced sufficiently on this level of the F-reagent content so that little influence was caused by the amount of PAE in the range of $0.3 \%-1.2 \%$. When PAE added was increased up to $0.6 \%$, sizing degree was particularly improved. But the excess PAE added to the slurry rather damaged liquid-repellency. The F-reagent content in the handsheets showed the tendency similar to that of water-repellency.

With respect to the adsorption of PAE to pulp fibers, several studies reported that almost all the amount of PAE added to the furnish was adsorbed to pulp fibers when the addition level was below ca. $2 \%$ (on dry weight pulp) $[6,7]$. But in our study it should be considered that the pulp slurry was far more dilute $(0.15 \%)$ than in those studies $(2 \%$ or $4 \%)$ and that the time of stirring the 
slurry between the addition of PAE and the F-reagent was as short as $1 \mathrm{~min}$. Therefore a part of PAE may be present in the slurry without attaching to pulp fibers at the time of addition of the F-reagent. Thus, the excess PAE probably prevented the F-reagent from retaining in handsheets.

Another remarkable phenomenon was that obvious differences in sizing degrees were observed between the handhsheets, although the F-reagent contents were almost the same; between handsheets prepared with $0.1 \%$ and $1.2 \% \mathrm{PAE}$, and between those prepared with $0.3 \%$ and $0.6 \%$ PAE (see Fig. 4). From these results, PAE was proved to be one of the important factors for water-repellency of handsheets prepared with the F-reagent. Similar results were observed also in the following section, in which the addition sequence of additives was changed from PAE $\rightarrow$ the F-reagent to the F-reagent $\rightarrow$ PAE. This effect is to be discussed later.

\subsection{Influences of the order of addition of the additives to pulp slurry}

When the F-reagent and PAE were added in the inverse sequence as in the case of Fig. 3, degrees of liquid-repellency and the F-reagent content decreased (Fig. 5). Thus, to improve liquid-repellency of the handsheets, it is essential to form cationic sites on anionic pulp fibers in the pulp slurry before adding the F-reagent to the pulp slurry. This result is inconsistent with the case for rosin soap size-alum sizing systems, where higher sizing levels can be achieved when rosin soap size, i.e. water-soluble rosin acid potassium salt, is first added to a pulp slurry.

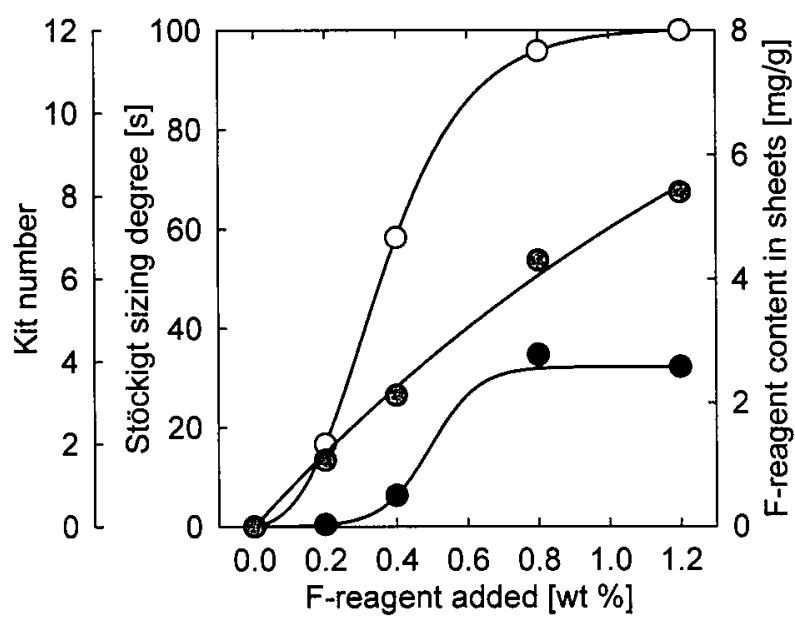

Size :- Kit:O F-reagent content:

Fig. 5 Water- and oil-repellency and F-reagent content of the handsheets prepared with F-reagent $\rightarrow$ $0.3 \%$ PAE. The handsheets were prepared after stirring the slurry for $1 \mathrm{~min}$, and heated at $105^{\circ} \mathrm{C}$ for $30 \mathrm{~min}$ after conditioning.
Thus, the system of PAE and the F-reagent is essentially different from that of the rosin soap size and alum.

Another characteristic phenomenon was that addition sequence of additives induced clear differences in liquid-repellency between handsheets containing similar amounts of the F-reagent. For instance, the handsheets prepared with $1.2 \%$ F-reagent in Fig. 5 had a roughly equal F-reagent content to that of the handsheets prepared with $0.8 \%$ F-reagent in Fig. 3, but the sizing degree of the former was smaller than that of the latter. From this result, it was concluded that the differences in liquid-repellency of the handsheets between the two addition sequences, the F-reagent $\rightarrow$ PAE and PAE $\rightarrow$ the F-reagent, could not be explained in terms of the retention of the F-reagent. There could be other factors such as agglomeration and/or distribution of the F-reagent components in the handsheets, that may have brought about such different liquid-repellency of the handsheets with the similar F-reagent content.

\subsection{Influences of stirring time of the pulp slurry before papermaking}

When stirring time of the pulp slurry after the addition of $0.8 \%$ F-reagent following $0.3 \%$ PAE was extended from $1 \mathrm{~min}$ to $5,10,30 \mathrm{~min}$ and to $1,2,4,8,12$, and $24 \mathrm{~h}$, degrees of both water- and oil-repellency clearly decreased (Fig. 6). The F-reagent contents in the handsheets decreased up to the stirring time of $4 \mathrm{~h}$. However, from 4 to $12 \mathrm{~h}$ the F-reagent content slightly increased, whereas liquid-repellency of the handsheets never recovered. This clearly negative effect of extended stirring time has rarely

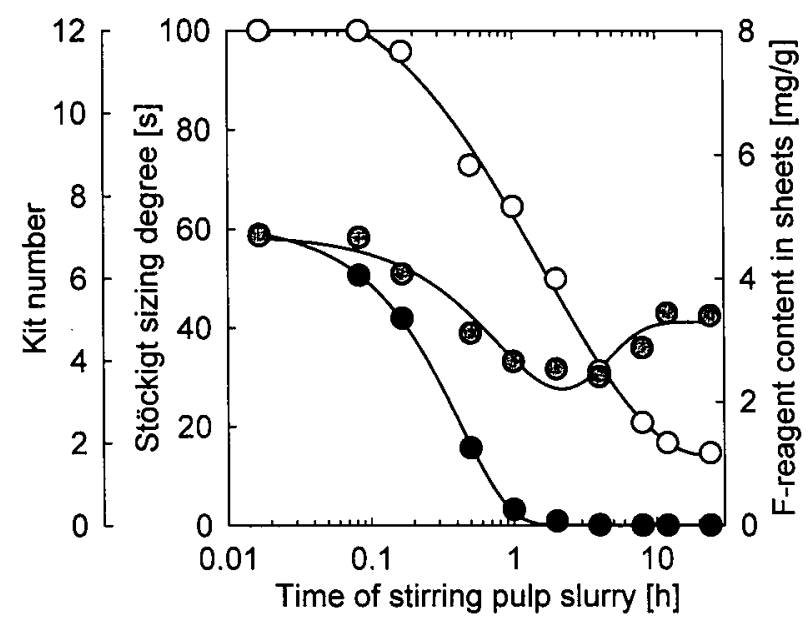

Size :- Kit:O F-reagent content :-

Fig. 6 Water- and oil-repellency and F-reagent content of the handsheets prepared from slurries stirred for various time after addition of $0.3 \%$ PAE $\rightarrow$ $0.8 \%$ F-reagent. The handhseets were heated at $105^{\circ} \mathrm{C}$ for $30 \mathrm{~min}$ after conditioning. 
been observed in AKD, ASA and rosin sizing systems. [8]

\subsection{Influences of heating temperature of hand- sheets after conditioning}

The heat treatment of the handsheets clearly improved their water-repellency. Having been prepared from the pulp slurry stirred for $1 \mathrm{~min}$ after the addition of $0.3 \%$ PAE and $0.8 \%$ F-reagent in this sequence, the handsheets were heated for $30 \mathrm{~min}$ at $40,60,80,105,120$ and $150^{\circ} \mathrm{C}$ after the drying for more than 1 day at room temperature. Under these conditions sizing degree increased especially from $60^{\circ} \mathrm{C}$ to $120^{\circ} \mathrm{C}$ (Fig. 7). On the other hand, oil-repellency showed little increases or maintained a constant value because oil-repellency of the handsheets had already reached to the maximum value without heating treatment.

When the heating temperature was raised from $120^{\circ} \mathrm{C}$ to $150^{\circ} \mathrm{C}$, water-repellency slightly decreased. Yet, within the range of these temperatures, thermal treatment could not lead to decomposition of the components of handsheets such as cellulose $[9,10]$. Thus, this phenomenon of the decrease in water-repellency may be due to thermal decomposition of PAE. With regard to the F-reagent used in this study, the contents in the handsheets were scarcely changed at 40,105 and $150^{\circ} \mathrm{C}$, and thus it was suggested that the F-reagent was not volatile during the thermal treatment within the range of these temperatures.

\subsection{Factors influencing liquid-repellency of handsheets}

All the results obtained so far in this study show that oil-repellency is roughly governed by the F-reagent con-

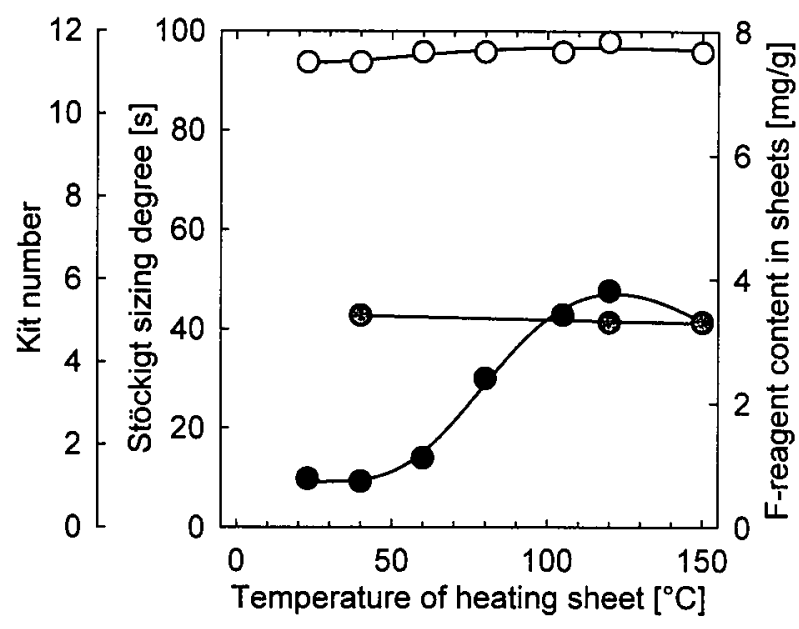

Size:- Kit:O F-reagent content :-

Fig. 7 Water- and oil-repellency and F-reagent content of the handsheets heated for $30 \mathrm{~min}$ at various temperatures after conditioning. The handsheets were prepared after stirring the slurry for $1 \mathrm{~min}$ after addition of $0.3 \% \mathrm{PAE} \rightarrow 0.8 \% \mathrm{~F}$-reagent.

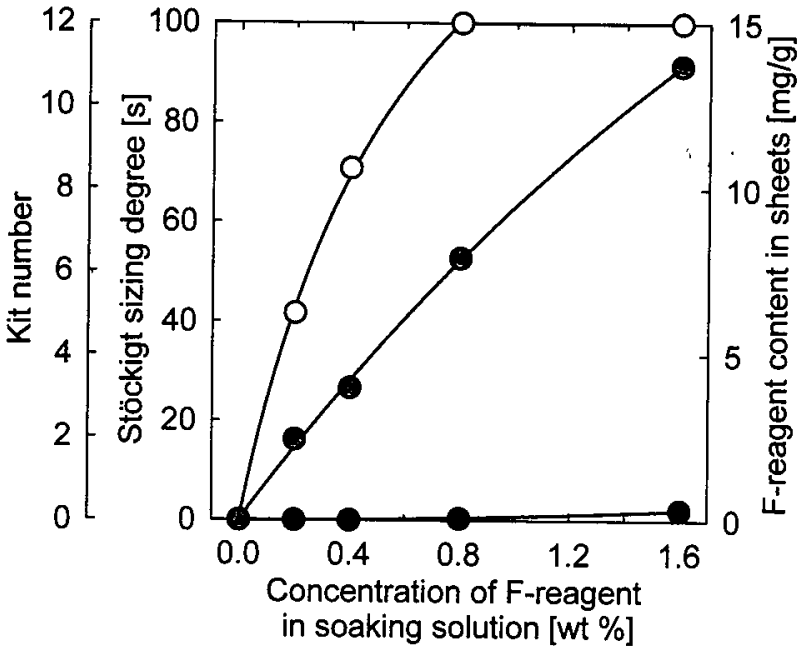

Size :- Kit:O F-reagent content:

Fig. 8 Water- and oil-repellency and F-reagent content of handsheets soaked in F-reagent solutions for $10 \mathrm{~s}$ without PAE. The handsheets were heated at $105^{\circ} \mathrm{C}$ for $30 \mathrm{~min}$.

tents in the handsheets or retention values of the F-reagent. On the other hand, sizing degree is sensitively influenced by handsheet-making conditions and behavior of water-repellency cannot be explained only in terms of the F-reagent content.

Fig. 8 shows water- and oil-repellency of the handsheets prepared by soaking in 0-1.6\% F-reagent solutions for $10 \mathrm{~s}$ followed by pressing and drying. The base handsheets were prepared beforehand from the pulp slurry without any additives. In the case of this soaking treatment, sizing degrees were extremely low even though sufficient amounts of the F-reagent were retained in the handsheets. On the other hand, high degrees of oil-repellency appeared on the handsheets, although the efficiency of oil-repellency was lower than that for the internal addition. Critical surface tension of oil is generally smaller than that of water so that water-repellency should appear on the handsheets more distinctively than oil-repellency according to the wetting theory. Thus, the phenomenon observed above is inconsistent with this theory.

One of the probable explanations for this discrepancy between the predicted and obtained results is that hydrophilicity due to phosphate groups of the F-reagent molecules overcomes hydrophobicity due to perfluoroalkyl groups. The phosphate groups must be present in the F-reagent molecules to maintain their water-solubility and to avoid agglomeration of the F-reagent molecules in the pulp slurry during the papermaking process, where water is always present. On the other hand, the presence 

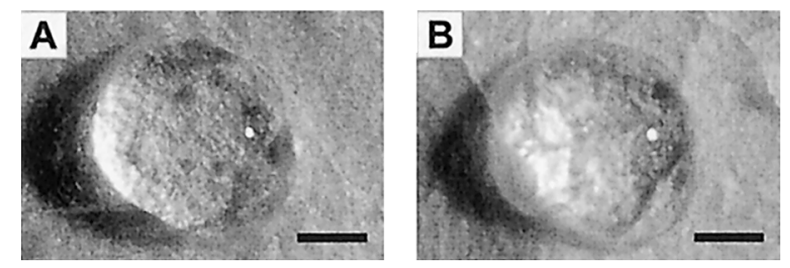

Fig. 9 Optical microphotographs of precipitates prepared from a F-reagent solution by adding PAE, on which droplets of water (A) or salad oil (B) were put. Each scale bar corresponds to $1 \mathrm{~mm}$.

of phosphate groups may have nearly no influence on oil-repellency, which is governed more simply by quantity of the perfluoroalkyl groups in the handsheets. Thus, conversion of the hydrophilic phosphate groups to hydrophobic ones by some structural changes of the F-reagent in handsheets may be required to improve water-repellency of the handsheets.

When a diluted F-reagent solution was poured into PAE solutions, precipitates appeared probably by formation of water-insoluble ion-complexes of the F-reagent and PAE. After drying of this precipitate in vacuo at $80^{\circ} \mathrm{C}$ for $3 \mathrm{~h}$, water- and oil-repellency were observed by putting water or salad oil droplets on the precipitates (Fig. 9). The precipitates showed clear water- and oil-repellency to a certain degree. Because nearly no F-reagent components were retained in the handsheets without the cationic retention aid PAE, the F-reagent components retained in the handsheets with PAE are likely to have chemical structures almost equal to those obtained as the precipitates in Fig. 9. Nevertheless, as shown in the former sections, it seems difficult to bring about water-repellency effectively on the handsheets prepared by internal addition of the F-reagent and PAE.

At this moment, details about the reasons for the low water-repellency of the handsheets prepared with the F-reagent are unknown. Agglomeration behavior of the hydrophobic F-reagent components formed in the handsheets may have influenced the resultant water-repellency. SEM analysis combined with EDX mapping techniques are now applied to make clear the agglomeration behavior or distributions of the F-reagent components in the handsheets prepared under various conditions.

\section{Conclusions}

With regard to appearance of water- and oil-repellency of handsheets prepared by internal addition of the fluo- rine-containing reagent, the following conclusions were obtained.

- X-ray fluorescence method was appropriate for a rough determination of the F-reagent content in handsheets, whereas the flask combustion method was apt for an exact determination of that content.

- The phosphate-type fluorine-containing reagent used in this study is retained in handsheets by electrostatic interactions through the cationic polymer PAE. Without PAE, nearly no F-reagent was retained in the handsheets.

- An excess addition of PAE brings about a decrease of the F-reagent content and liquid-repellency of the handsheets.

-While oil-repellency is roughly governed by the F-reagent content in the handsheets, water-repellency of the handsheets is sensitively influenced by the handsheet-making conditions; the order of addition of the additives into pulp slurries, the stirring time of pulp slurries after addition of the F-reagent, the heat treatment after conditioning the handsheets and so on.

- Not only retaining the F-reagent but also controlling distribution or agglomeration of the F-reagent components in the handsheets is probably the key factor for the improvement of water-repellency.

\section{References}

1. L. Yang, R. Pelton, F. McLellan and M. Fairbank, Tappi J., 82(9), 128 (1999).

2. JIS Method, "Pulps-Preparation of laboratory sheets for physical testing", P8222 (1998).

3. JIS Method, "Testing Method for Stöckight Sizing Degree of Paper", P8122 (1976).

4. TAPPI Test Method, "Grease resistance test for paper and paperboard", T 599 pm-96, TAPPI Press (1996).

5. M. Ishikawa, private communication.

6. D. I. Devore, N. S. Clungeon and S. A. Fischer, ACS Sym. Ser, 548, 394 (1994).

7. D. I. Devore, N. S. Clungeon and S. A. Fischer, Tappi $J ., 74(12), 135$ (1991).

8. K. Ohno, A. Isogai and F. Onabe, J. Wood Sci., 45, 481 (1999).

9. F. Shafizadeh, Advances Carbohydr. Chem., 23, 419 (1968).

10. F. Shafizadeh and A. G. W. Bradbury, J. Appl. Polym. Sci., 23, 1431 (1979). 